\title{
Taylor expansion method for linear lattice Boltzmann schemes with an external force Application to boundary conditions
}

\author{
François Dubois $^{a b}$ Pierre Lallemand $^{c}$ and Mohamed Mahdi Tekitek ${ }^{d}$ \\ ${ }^{a}$ Conservatoire National des Arts et Métiers, Paris, France, \\ Laboratoire de Mécanique des Structures et des Systèmes Couplés. \\ ${ }^{b}$ Department of Mathematics, University Paris-Sud, \\ Bât. 425, F-91405 Orsay Cedex, France. \\ ${ }^{c}$ Beijing Computational Science Research Center, \\ Beijing Run Ze Jia Ye, China. \\ ${ }^{d}$ University Tunis El Manar, Faculty of Sciences, Tunis, Tunisia. \\ francois.dubois@math.u-psud.fr, pierre.lallemand1@free.fr, mohamedmahdi.tekitek@fst.rnu.tn
}

10 december $2013 *$

\begin{abstract}
In this contribution we show that it is possible to get the macroscopic fluid equations of a lattice Boltzmann scheme with an external force using the Taylor expansion method. We validate this general expansion by a detailed analysis of the boundary conditions. We derive "quartic parameters" that enforce the precision of the boundary scheme. We explicit and validate the corresponding relations for a Poiseuille flow computed with the D2Q13 lattice Boltzmann scheme.
\end{abstract}

Keywords: bounce-back, adiabatic wall, acoustics, Poiseuille flow.

AMS classification: $6505,76 \mathrm{~N} 15,80 \mathrm{~A} 20,82 \mathrm{C} 20$.

* Contribution published in the proceedings of "High Order Nonlinear Numerical Schemes for Evolutionary PDEs", Lecture Notes in Computational Science and Engineering, volume 99, pages 89-107, Rémi Abgrall et al. Editors, Springer, 2014. Edition 02 december 2014. 


\section{Introduction}

- The lattice Boltzmann scheme is a mesoscopic method. It deals with a small number of functions that can be interpreted as populations of fictitious particles. It has been developed more than 20 years ago by Qian et al. [19], Succi et al. [20] among others. This method can simulate various fluid dynamics problems. The dynamics of those particles is such that time, space and momentum are discretized. They move at successive discrete times between the nodes of a regular square lattice. The velocity space is discretized by a reduced set of discrete velocities. The unknown is the distribution $\left\{f_{i}\right\}$ which is function of velocities, space location and discrete time. In each time step there are two fundamental steps : advection and collision. The advection step is linear and allows the connection of a given vertex with its neighbors. It corresponds to the characteristic method with a Courant number exactly equal to one. The collision step is nonlinear and local in space. The discrete dynamics solve a system of differential equations that converge to an equilibrium state for large time (see the details e.g. in [5]).

- A theoretical analysis of the lattice Boltzmann scheme was proposed by Qian et al. [19] and d'Humières [11] with a Chapmann Enskog expansion coming from statistical physics. We refer to Asinari and Ohwada [1] for a method of analysis based on the Grad moment system. A fruitful idea developed by Junk et al [13] and our team [5] is to use the so-called equivalent equation method derived by Lerat-Peyret [15] and Warming-Hayett [21] in the context of classical finite difference schemes. The time step is considered as an infinitesimal parameter and the finite differences are expanded into a family of equivalent partial differential operators. We observe that the emerging viscosity with the lattice Boltzmann method is in some sense a discrepancy of the scheme that can explicited.

- The main goal of this study is to extend the linearized analysis of a lattice Boltzmann scheme using the Taylor expansion method $[2,5]$ to the case where there is an external body force. In fact few methods $[4,9,16,17,18]$ have been used to incorporate external forces in lattice Boltzmann scheme. In this study, a fraction $\theta$ (with $0 \leq \theta \leq 1$ ) of the force term is added before the collision step and a fraction $(1-\theta)$ just after. Usually $\theta$ is equal to $\frac{1}{2}$. In this contribution we consider a given external force variable in time and space.

- Boundary conditions are performed to impose a given velocity (or a given pressure) on a part of the physical boundary. These conditions have to be translated in terms of the lattice Boltzmann scheme. When the scheme is completely defined for internal vertices a numerical boundary conditions of bounce-back or anti-bounce-back type (see e.g. Bouzidi et al. [3]) is equivalent to impose a zero value for some combination of the particle distributions. With the help of the Taylor expansion, it is possible to make in evidence the errors associated to several methods for one dimensional thermal problems or two dimensional fluid problem. This defines so-called "quartic parameters" (see [6]). For the general framework of this kind of methodology, we refer to Ginzburg and Adler [7], Ginzburg et al. [8] and Ginzburg and d'Humières [12]. Our approach is to extend our previous work [6] to three representative examples. In particular a precise choice of relaxation rates allows to spectacularly decrease the error associated to the 
scheme. In the case of the Poiseuille flow the numerical evaluation of the analytical solution can even be possible with this methodology.

- In the first section, we precisely define our numerical scheme. In section 2, we extend the linearized analysis of a lattice Boltzmann scheme using the Taylor expansion method [2]. In section 3 , we study a very simple one dimensional thermal problem with a given temperature on the boundary. The existence of the source term allows an experimental validation of our methodology applied to the boundary conditions. In section 4, we study a two dimensional scheme with the D2Q9 scheme in the context of the Poiseuille flow. This flow is considered with an external force term instead of physical pressure term. Theoretical analysis allows to propose quartic values for the relaxation coefficients and for the parameter $\theta$. These parameters are confronted to a numerical test for this model with 13 velocities in section 5. To our knowledge this kind of determination and validation has not been proposed by other teams previously.

\section{1) Lattice Boltzmann scheme with a forcing drift}

- In the following, the notation " $\mathrm{D} d \mathrm{Q} q$ " denotes a lattice Boltzmann scheme with $d$ space dimensions and $q$ velocities. Space is discretized by a regular lattice $\mathscr{L}$ parametrized by a spatial scale $\Delta x$. The time step is denoted by $\Delta t$. A numerical celerity $\lambda$ is naturally defined by $\lambda \equiv \frac{\Delta x}{\Delta t}$. The $q$ discrete velocities can be written as $v_{j}=\lambda e_{j}$ for $0 \leq j \leq q-1$. A classical example is given by the D2Q9 scheme illustrated on the Figure 1.

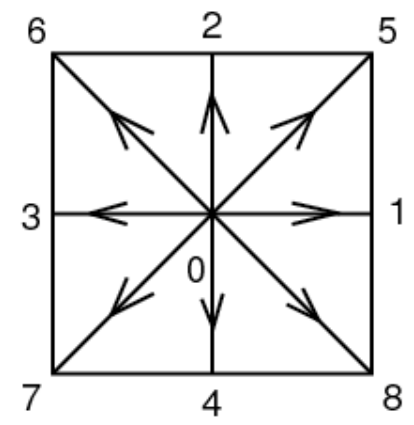

Figure 1. Stencil for the D2Q9 lattice Boltzmann scheme.

- The lattice Boltzmann scheme is formulated in both spaces of particles and moments $m_{k}$, as proposed by d'Humières [11]. We introduce an invertible matrix

$$
M=\left(M_{k j}\right), \quad 0 \leq k, j \leq q-1 .
$$

Then the moments $m_{k}$ for $0 \leq k \leq q-1$ are defined by linearity:

$$
m_{k} \equiv \sum_{j=0}^{q-1} M_{k j} f_{j}
$$

The moment vector $m \in \mathbb{R}^{q}$ can be written as

$$
m=\left(\begin{array}{c}
V \\
Y
\end{array}\right)
$$


with two kinds of quantities: conserved moments $V \in \mathbb{R}^{N}$ and slave moments $Y \in \mathbb{R}^{q-N}$. The conserved moments $V$ are not affected by the collision step when there is no forcing term. If $N=1$ there is exactly one scalar partial differential equation and when $N>1$ we obtain an underlying system of $N$ partial differential equations. The family of slave moments $Y$ relax during the collision step towards an equilibrium distribution. An elementary time evolution of a lattice Boltzmann scheme with a force is composed by four steps (see Guo et al. [9]) : computation of the dynamical variables, collision (through simple relaxation), forcing of the conserved moments and advection.

- The computation of the dynamical variables $W$ from the first component $V$ of the moments (1), is given by:

$$
W=V+\theta \Delta t F
$$

where $\theta$ is a fixed scalar in $[0,1]$ and $F \in \mathbb{R}^{N}$ is a given force term. Remark that the postprocessing is obtained from the knowledge of these dynamical variables.

- The collision and relaxation step is a redistribution of the populations $\left\{f_{i}\right\}$ at each node $x$. The $N$ first moments $W$ are not affected by the relaxation. The other "non-conserved" moments relax with a time constant $\tau_{k}$ towards the equilibrium values $m_{k}^{e q}$. In this contribution the equilibrium values are a linear function of the dynamical variables $W$. We suppose that the equilibrium values $Y^{e q}$ of the non-conserved moments are linear function of dynamical variables. We have

$$
Y^{e q}=E W
$$

where $E$ is a fixed matrix with $q-N$ lines and $N$ columns. Thus the relaxation is simply described by an ordinary differential equation:

$$
\frac{\mathrm{d}}{\mathrm{dt}}\left(m_{k}-m_{k}^{e q}\right)+\frac{1}{\tau_{k}}\left(m_{k}-m_{k}^{e q}\right)=0, \quad \text { for } \quad k \geq N .
$$

Using an explicit first order Euler scheme, we obtain the algorithm:

$$
m_{k}^{*}=\left(1-s_{k}\right) m_{k}+s_{k} m_{k}^{e q},
$$

where the superscript $*$ indicates "post-collision" state. The relaxation rates $s_{k} \equiv \frac{\Delta t}{\tau_{k}}$ have to satisfy $0<s_{k}<2$ in order to maintain stability of the scheme. With the notation (1) the relaxation step is performed in the moment space as follows:

$$
Y^{*}=(\mathrm{Id}-S) Y+S Y^{e q},
$$

where $S$ is the diagonal matrix of the relaxation times $s_{k}$ for $k \geq N$. We remark that this collision step is local in space.

- Due to the force term, the conserved moments during the collision step evolve according to

$$
V^{*}=V+\Delta t F=W+(1-\theta) \Delta t F
$$


If ()$^{t}$ denotes the transpose of the matrix $(\quad)$, the moments $m^{*} \equiv\left(V^{*}, Y^{*}\right)^{\mathrm{t}}$ after collision are determined by the relations (5) and (6). After the forcing and collision steps, the particle distribution $f_{j}^{*}$ is recovered by the inversion of a linear system of small size:

$$
f_{j}^{*}=\sum_{k=0}^{q-1} M_{j k}^{-1} m_{k}^{*}, \quad 0 \leq j \leq q-1 .
$$

- The advection step corresponds to a method of characteristics with a Courant number equal to 1 for the advection with velocity $v_{j}$. We assume here that for each node $x$ and each velocity $v_{j}$, the vertex $x-v_{j} \Delta t$ is also a node of the lattice. The particles moves from a lattice node $x$ to either itself (for $v_{0}=0$ ) or one of the $q-1$ neighbors $x_{j}=x+v_{j} \Delta t$ for velocities $v_{j} \neq 0$ as presented in the Figure 1. So a time step of a lattice Boltzmann scheme can be written as:

$$
f_{i}(x, t+\Delta t)=f_{i}^{*}\left(x-v_{i} \Delta t, t\right), \quad 0 \leq i \leq q-1, x \in \mathscr{L},
$$

where $f_{i}^{*}$ denotes the post-collision population of particles evaluated at the end (7) of the previous step.

\section{2) Taylor expansion method for the equivalent equations}

- In this contribution, we extend the "Berliner version" [2] of the Taylor expansion method in order to derive macroscopic equivalent equations when an external forcing term is present. We suppose on one hand that the dynamical variables $W$ satisfy a partial differential equation:

$$
\partial_{t} W=\alpha_{1} W+\Delta t \alpha_{2} W+\Delta t^{2} \alpha_{3} W+\cdots+\gamma_{0} F+\Delta t \gamma_{1} F+\ldots
$$

and on the other hand that the non conserved moments $Y$ follow a dynamical expansion of the type

$$
Y=E W+\Delta t \beta_{1} W+\Delta t^{2} \beta_{2} W+\cdots+\Delta t \rho_{0} F+\Delta t^{2} \rho_{1} F+\ldots
$$

In the equations (9) and (10), $\alpha_{j}, \gamma_{j}, \beta_{j}$ and $\rho_{j}$ are space derivative operators of order $j$. Moreover $\alpha_{j}$ and $\gamma_{j}$ are $N \times N$ matrices and $\beta_{j}$ and $\rho_{j}$ are $(q-N) \times N$ matrices. Note here that the equation (9) is a system of $N$ partial differential equations that represent the evolution of the conserved variables. It gives the macroscopic (equivalent) equation of the physical problem. The equation (10) describes the expansion of the "slave variables" $Y$ in terms of the conserved quantities. Due to (5), we first write the collision step as follows:

$$
Y^{*}=(\mathrm{Id}-S) Y+S Y^{e q}=(\operatorname{Id}-S) Y+S E W
$$

due to (3). Then, according to the relation (2), we have

$$
Y^{*}=(\operatorname{Id}-S) Y+S E(V+\Delta t \theta F)=S E V+(\operatorname{Id}-S) Y+\Delta t \theta S E F .
$$

- Then the moments $m^{*}$ after forcing and relaxation can be presented as follows:

$$
m^{*}=\left(\begin{array}{c}
V^{*} \\
Y^{*}
\end{array}\right)=\left(\begin{array}{cc}
I & 0 \\
S E & I-S
\end{array}\right)\left(\begin{array}{c}
V \\
Y
\end{array}\right)+\Delta t\left(\begin{array}{c}
I \\
\theta S E
\end{array}\right) F .
$$


Secondly, we rewrite the scheme (8) in moment space:

$$
\left(\begin{array}{l}
V \\
Y
\end{array}\right)_{k}(x, t+\Delta t)=m_{k}(x, t+\Delta t)=\sum_{j, l} M_{k, j} M_{j, l}^{-1} m_{l}^{*}\left(x-v_{j} \Delta t, t\right)
$$

then

$$
\left(\begin{array}{c}
V \\
Y
\end{array}\right)_{k}(x, t+\Delta t)=\sum_{j, l, \mu} M_{k, j} M_{j, l}^{-1}\left(-v_{j}\right)^{\mu} \frac{\Delta t^{|\mu|}}{\mu !} \partial_{\mu} m_{l}^{*}(x, t) .
$$

In the previous relation $\partial_{\mu}$ is a space derivation of order $\mu=\left(\mu_{1}, \mu_{2}, \ldots, \mu_{d}\right) \in \mathbb{N}^{d}$ and $\mu !=\mu_{1} ! \ldots \mu_{N} !$. The length $|\mu|$ of the multi-index $\mu$ is equal to $\sum_{j} \mu_{j}$. We inject the value (11) of $m^{*}$ in the previous expression. We arrange the previous formal series as increasing powers of $\Delta t$. We obtain the following equation:

$$
\left(\begin{array}{c}
V \\
Y
\end{array}\right)(t+\Delta t)=\sum_{n \geq 0} \Delta t^{n}\left(\begin{array}{cc}
A_{n} & B_{n} \\
C_{n} & D_{n}
\end{array}\right)\left(\begin{array}{c}
V \\
Y
\end{array}\right)(t)+\sum_{n \geq 0} \Delta t^{n+1}\left(\begin{array}{c}
G_{n} \\
H_{n}
\end{array}\right) F .
$$

In (13), the matrices $A_{n}, B_{n}, C_{n}, D_{n}, G_{n}$ and $H_{n}$ are space derivation operators of order $n$. They are easy to identify with the help of the right hand side of the equation (12). The order zero of the development (13) relative to $\Delta t$ is deduced from (11) by identification:

$$
\left(\begin{array}{cc}
A_{0} & B_{0} \\
C_{0} & D_{0}
\end{array}\right)=\left(\begin{array}{cc}
I & 0 \\
S E & \mathrm{I}-S
\end{array}\right), \quad\left(\begin{array}{c}
G_{0} \\
H_{0}
\end{array}\right)=\left(\begin{array}{c}
\mathrm{I} \\
\theta S E
\end{array}\right) .
$$

- Proposition 1. Taylor expansion at order 1.

The equivalent equations and the non conserved moments for linear lattice Boltzmann scheme with external force up to first order are given by the relations

$$
\partial_{t} W=\alpha_{1} W+\gamma_{0} F+\mathrm{O}(\Delta t), \quad Y=E W+\Delta t\left(\beta_{1} W+\rho_{0} F\right)+\mathrm{O}\left(\Delta t^{2}\right)
$$

with

$$
\left\{\begin{array}{l}
\alpha_{1}=A_{1}+B_{1} E, \quad \beta_{1}=S^{-1}\left(C_{1}+D_{1} E-E \alpha_{1}\right), \\
\gamma_{0}=G_{0}=\mathrm{I}, \quad \rho_{0}=S^{-1}\left(H_{0}-E \gamma_{0}-\theta S E\right) .
\end{array}\right.
$$

\section{Proof of Proposition 1.}

We have from (13) :

$$
\begin{aligned}
V(t+\Delta t) & =V+\Delta t \partial_{t} V+\mathrm{O}\left(\Delta t^{2}\right) \\
& =V+\Delta t\left(A_{1} V+B_{1} Y\right)+\Delta t G_{0} F+\mathrm{O}\left(\Delta t^{2}\right)
\end{aligned}
$$

and due to (14),

$$
\begin{aligned}
Y(t+\Delta t) & =Y+\Delta t \partial_{t} Y+\mathrm{O}\left(\Delta t^{2}\right) \\
& =(\mathrm{Id}-S) Y+S E V+\Delta t\left(C_{1} V+D_{1} Y\right)+\Delta t H_{0} F+\mathrm{O}\left(\Delta t^{2}\right)
\end{aligned}
$$

Using the relation (2) we have

$$
V=W-\theta \Delta t F
$$


and the development at equilibrium (order zero)

$$
Y=E W+\mathrm{O}(\Delta t)
$$

we obtain on one hand

$$
\partial_{t} W=\left(A_{1}+B_{1} E\right) W+G_{0} F+\mathrm{O}(\Delta t)
$$

and on the other hand

$$
Y=E W+\Delta t S^{-1}\left[C_{1}+D_{1} E-E\left(A_{1}+B_{1} E\right) W\right]+\left(H_{0}-E \gamma_{0}-\theta S E\right) F+\mathrm{O}\left(\Delta t^{2}\right) .
$$

By identification of the previous relations with the ansatz (9)(10), we obtain the relations (15) at first order with the coefficients $\alpha_{1}, \beta_{1}, \gamma_{0}$ and $\rho_{0}$ explicited in the relations (16).

\section{- Proposition 2. Taylor expansion at order 2.}

The equivalent equations for linear lattice Boltzmann scheme with external force, as described in first section, up to second order, are:

$$
\left\{\begin{aligned}
\partial_{t} W & =\alpha_{1} W+\gamma_{0} F+\Delta t\left(\alpha_{2} W+\gamma_{1} F\right)+\mathrm{O}\left(\Delta t^{2}\right) \\
Y & =E W+\Delta t\left(\beta_{1} W+\rho_{0} F\right)+\Delta t^{2}\left(\beta_{2} W+\rho_{1} F\right)+\mathrm{O}\left(\Delta t^{3}\right)
\end{aligned}\right.
$$

with coefficients $\alpha_{1}, \beta_{1}, \gamma_{0}$ and $\rho_{0}$ proposed in (16) and

$$
\left\{\begin{array}{c}
\alpha_{2}=B_{1} \beta_{1}+A_{2}+B_{2} E-\frac{1}{2} \alpha_{1}^{2} \\
\gamma_{1}=B_{1} \rho_{0}+G_{1}-\theta A_{1}-\frac{1}{2}\left[\alpha_{1} \gamma_{0}+\left(\gamma_{0}-2 \theta\right) \partial_{t}\right] \\
\beta_{2}=S^{-1}\left[D_{1} \beta_{1}-E \alpha_{2}-\beta_{1} \alpha_{1}-E \frac{\alpha_{1}^{2}}{2}+C_{2}+D_{2} E\right] \\
\rho_{1}=S^{-1}\left[D_{1} \rho_{0}+H_{1}-E \gamma_{1}-\beta_{1} \gamma_{0}-\frac{1}{2} E \alpha_{1} \gamma_{0}\right. \\
\left.-\frac{1}{2} E \gamma_{0} \partial_{t}-\rho_{0} \partial_{t}-\theta C_{1}\right] .
\end{array}\right.
$$

\section{Proof of Proposition 2.}

We first differentiate relative to time the first relation of (15):

$$
\begin{aligned}
\partial_{t}^{2} W & =\partial_{t}\left(\alpha_{1} W+F\right)+\mathrm{O}(\Delta t)=\alpha_{1}\left(\alpha_{1} W+F\right)+\partial_{t} F+\mathrm{O}(\Delta t) \\
& =\alpha_{1}^{2} W+\left(\alpha_{1}+\partial_{t}\right) F+\mathrm{O}(\Delta t) .
\end{aligned}
$$

We can therefore develop the dynamical variables $W$ at time $t+\Delta t$ up to second order. Due to the definition (2), we have:

$$
\begin{aligned}
W(t+\Delta t)= & V(t+\Delta t)+\theta \Delta t F(t+\Delta t) \\
= & V(t)+\Delta t\left(A_{1} V(t)+B_{1} Y(t)\right)+\Delta t^{2}\left(A_{2} V(t)+B_{2} Y(t)\right) \\
& +\theta \Delta t F(t+\Delta t)+\mathrm{O}\left(\Delta t^{3}\right) \\
= & W(t)-\theta \Delta t F(t)+\theta \Delta t F(t+\Delta t)+\Delta t\left(A_{1}(W-\theta \Delta t F)\right. \\
& \left.+B_{1}\left(E W+\Delta t \beta_{1} W+\Delta t \rho_{0} F\right)\right)+\Delta t^{2}\left(A_{2} W+B_{2} E W\right)+\mathrm{O}\left(\Delta t^{3}\right) .
\end{aligned}
$$


Then taking into account the Taylor formula at second order accuracy and the expression of $\partial_{t}^{2} W$ :

$$
\begin{aligned}
W+\Delta t \partial_{t} W+ & \frac{\Delta t^{2}}{2}\left[\alpha_{1}^{2} W+\left(\alpha_{1}+\partial_{t}\right) F\right]+\mathrm{O}\left(\Delta t^{3}\right) \\
= & W+\theta \Delta t^{2} \partial_{t} F+\Delta t\left(A_{1} W+B_{1} E W\right) \\
& \quad+\Delta t^{2}\left(-\Delta t F+B_{1} \beta_{1} W+B_{1} \rho_{0} F+A_{2} W+B_{2} E W\right)+\mathrm{O}\left(\Delta t^{3}\right)
\end{aligned}
$$

After dividing by $\Delta t$ :

$$
\begin{aligned}
\partial_{t} W=\left(A_{1}+B_{1} E\right) W+F & +\Delta t\left[A_{2}+B_{2} E+B_{1} \beta_{1}-\frac{1}{2} \alpha_{1}^{2}\right] W \\
& +\Delta t\left[G_{1}+B_{1} \rho_{0}-\theta A_{1}-\frac{1}{2} \alpha_{1}+\left(\theta-\frac{1}{2}\right) \partial_{t}\right] F+\mathrm{O}\left(\Delta t^{2}\right)
\end{aligned}
$$

and the expressions of $\alpha_{2}$ and $\gamma_{1}$ in (18) are established.

To get the second relation of (17), we first look to the derivation relative to time of the nonconserved moments $Y$. We have

$$
\begin{aligned}
\partial_{t} Y= & \partial_{t}\left[E W+\Delta t\left(\beta_{1} W+\rho_{0} F\right)\right]+\mathrm{O}\left(\Delta t^{2}\right) \\
= & E\left[\begin{array}{l}
\left.\alpha_{1} W+F+\Delta t\left(\alpha_{2} W+\gamma_{1} F\right)+\mathrm{O}\left(\Delta t^{2}\right)\right] \\
\quad \\
\quad+\Delta t \beta_{1}\left(\alpha_{1} W+F\right)+\Delta t \rho_{0} \partial_{t} F+\mathrm{O}\left(\Delta t^{2}\right)
\end{array}\right. \\
= & E\left(\alpha_{1} W+F\right)+\Delta t\left[\left(E \alpha_{2}+\beta_{1} \alpha_{1}\right) W+\left(E \gamma_{1}+\beta_{1}+\rho_{1} \partial_{t}\right) F\right]+\mathrm{O}\left(\Delta t^{2}\right) .
\end{aligned}
$$

We differentiate relative to time at second order:

$$
\begin{aligned}
\partial_{t}^{2} Y & =\partial_{t}\left[E\left(\alpha_{1} W+F\right)\right]+\mathrm{O}(\Delta t)=E \alpha_{1}\left(\alpha_{1} W+F\right)+E \partial_{t} F+\mathrm{O}(\Delta t) \\
& =E \alpha_{1}^{2} W+\left(E \alpha_{1}+E \partial_{t}\right) F+\mathrm{O}(\Delta t)
\end{aligned}
$$

Then we have

$$
\begin{aligned}
S Y(t)= & S E V-(Y(t+\Delta t)-Y(t))+\Delta t\left(C_{1} V+D_{1} Y+\theta S E F\right) \\
& +\Delta t^{2}\left(C_{2} V+D_{2} Y+H_{1} F\right)+\mathrm{O}\left(\Delta t^{3}\right) \\
= & S E(W-\theta \Delta t F) \\
& -\Delta t\left[E\left(\alpha_{1} W+F\right)+\Delta t\left[\left(E \alpha_{2}+\beta_{1} \alpha_{1}\right) W+\left(E \gamma_{1}+\beta_{1}+\rho_{1} \partial_{t}\right) F\right]\right] \\
& -\frac{\Delta t^{2}}{2}\left[E \alpha_{1}^{2} W+\left(E \alpha_{1}+E \partial_{t}\right) F\right] \\
& +\Delta t\left[C_{1}(W-\theta \Delta t F)+D_{1}\left(E W+\Delta t \beta_{1} W+\Delta t \rho_{0} F\right)+\theta S E F\right] \\
& +\Delta t^{2}\left(C_{2} W+D_{2} E W+H_{1} F\right)+\mathrm{O}\left(\Delta t^{3}\right) \\
= & S\left[E W+\Delta t \beta_{1} W+\Delta t \rho_{0} F\right] \\
& +\Delta t^{2}\left[\left(C_{2}+D_{2} E+D_{1} \beta_{1}-\frac{1}{2} E \alpha_{1}^{2}-\left(E \alpha_{2}+\beta_{1} \alpha_{1}\right)\right) W\right. \\
& \left.+\left(H_{1}+D_{1} \rho_{0}-\theta C_{1}-\frac{1}{2}\left(E \alpha_{1}+E \partial_{t}\right)-\left(E \gamma_{1}+\beta_{1}+\rho_{0} \partial_{t}\right)\right) F\right]+\mathrm{O}\left(\Delta t^{3}\right) \\
&
\end{aligned}
$$

and the expressions of $\beta_{2}$ and $\rho_{1}$ proposed at the second line of the relations (18) are established because $\gamma_{0}$ is the identity matrix. 


\section{3) Boundary conditions for D1Q3 thermal problems}

- In general there is a difference between the assumed wall position and the "measured" one, where the numerical solution vanishes. For some particular lattice Boltzmann parameters, this difference is null up to order two on $\Delta x$. In this section, we study the impact of the knowledge given in the relations (17) (18) on the analysis of the boundary conditions for the lattice Boltzmann schemes. In general, the incoming particles are given as a simple function of the outgoing ones through specular reflection (bounce-back) [3] or specular "anti-reflection" (anti-bounceback) that are detailed in the corpus of the text. We first focus on the D1Q3 scheme for a scalar problem.

We introduce the following moment matrix $M$ for the D1Q3 (see Figure 2) lattice Boltzmann scheme:

$$
M=\left(\begin{array}{ccc}
1 & 1 & 1 \\
0 & \lambda & -\lambda \\
-2 \lambda^{2} & \lambda^{2} & \lambda^{2}
\end{array}\right)
$$

We consider only one conservation law $(N=1)$ and we set $\rho=W=f_{0}+f_{1}+f_{2}$. The equilibrium values $Y^{e q}$ of the two non conserved moments are given according to

$$
Y^{e q}=\left(\begin{array}{c}
0 \\
\lambda^{2} \\
\zeta
\end{array}\right) \rho \equiv E \rho .
$$

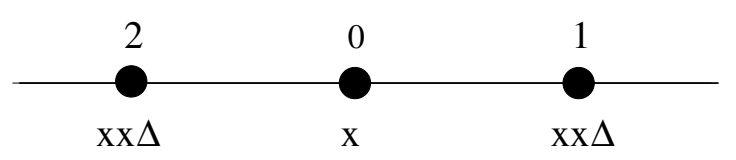

Figure 2. Stencil of the lattice Boltzmann scheme D1Q3.

- Proposition 3. Diffusion model with the D1Q3 lattice Boltzmann scheme.

With the previous lattice Boltzmann D1Q3 scheme, described by relations (2), (6), (8), (19) and (20), we have the equivalent equation

$$
\frac{\partial \rho}{\partial t}-\kappa \frac{\partial^{2} \rho}{\partial x^{2}}-F=\mathrm{O}\left(\Delta t^{2}\right)
$$

at second order for a stationary force $F$, with the thermal diffusivity

$$
\kappa=\frac{2+\zeta}{3} \Delta t \lambda^{2} \sigma_{1} \quad \text { with } \quad \sigma_{1} \equiv \frac{1}{s_{1}}-\frac{1}{2}
$$

\section{Proof of Proposition 3.}

There is only one scalar conservation and $W \equiv \rho$. According to the proposition 2 , we have the equivalent equation up to order two :

$$
\partial_{t} W=\alpha_{1} W+F+\Delta t\left(\alpha_{2} W+\gamma_{1} F\right)+\mathrm{O}\left(\Delta t^{2}\right) .
$$


The associated coefficients can be computed easily: $\alpha_{1}=0, \alpha_{2}=\lambda^{2} \sigma_{1} \frac{\zeta+2}{3}, \gamma_{0}=1$ and $\gamma_{1}=$ $\left(\theta-\frac{1}{2}\right) \partial_{t}$. We have in consequence $\gamma_{1} F=0$ if $\partial_{t} F=0$ and the equivalent equation is established.

- We consider the elementary one-dimensional Poisson problem with homogeneous Dirichlet boundary conditions:

$$
-\triangle u(x)=c \quad \text { for } 0<x<1, \quad u(0)=u(1)=0 .
$$

The solution of the problem (21) is quadratic. A uniform body force $F$ is considered to take into account the source term $c$ in the right hand side of the Poisson equation. We denote by $x_{b}$ the first node at the left in the domain ]0, 1[ and by $x_{e}$ a fictitious outside node (see Figure 3 ). The node $x_{b}$ is supposed to be located at a distance $\Delta q$ of the solid wall position $x_{i}$ (see Figure 3 ). We implement a so-called "anti-bounce-back" boundary condition to take into account the Dirichlet boundary conditions:

$$
f_{1}\left(x_{b}, t+\Delta t\right)=-f_{2}^{*}\left(x_{b}, t\right)+\Phi
$$

where $\Phi$ is a given boundary term directly related to the boundary data. Observe that this term is null if we consider e.g. homogenous Dirichlet boundary conditions.

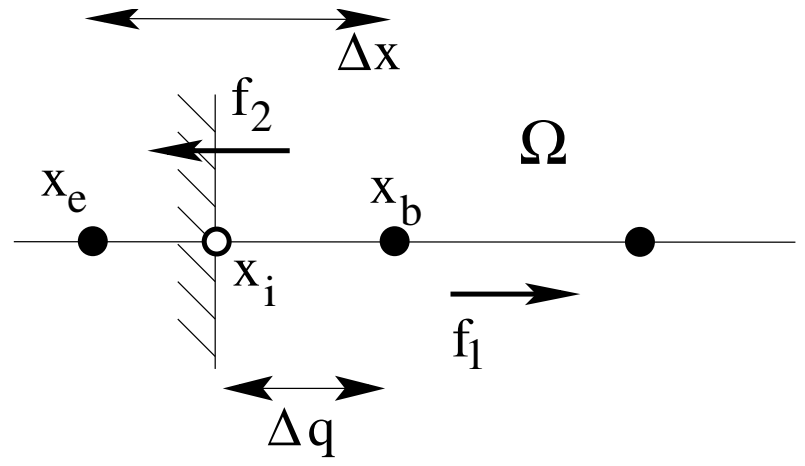

Figure 3. "Anti-bounce-back" numerical boundary condition for the D1Q3 lattice Boltzmann scheme. The boundary node $x_{b}$ is located at the distance $\Delta q$ of the theoretical boundary. The incoming particles $f_{1}\left(x_{b}\right)$ are determined from the knowledge of the outgoing density of particles $f_{2}\left(x_{b}\right)$.

- Proposition 4. Quartic treatment of a D1Q3 boundary condition.

For the above D1Q3 lattice Boltzmann scheme, we define

$$
\xi \equiv-3+8 \sigma_{1} \sigma_{2}-8 \sigma_{1}(1-2 \theta)
$$

The numerical solution of the Poisson problem (21) vanishes at third order formal accuracy for the position $\Delta q=\frac{\Delta x}{2}$ if the condition $\xi=0$ is satisfied.

\section{Proof of Proposition 4.}

Using the equation (10) on the non conserved moment, we have

$$
Y=E W+\Delta t \beta_{1} W+\Delta t^{2} \beta_{2} W+\Delta t \rho_{0} F+\Delta t^{2} \rho_{1} F .
$$


Then we perform a collision step to get

$$
Y^{*}=(\mathrm{I}-S) Y+S E Y+\Delta t \theta S E F .
$$

With the help of the inverse moments matrix $M^{-1}$, we deduce:

$$
f_{1}^{*}=\frac{1}{2 \lambda^{2}}\left[2 m_{2}^{*}+\lambda m_{1}^{*}\right] \quad \text { and } \quad f_{2}^{*}=\frac{1}{2 \lambda^{2}}\left[2 m_{2}^{*}-\lambda m_{1}^{*}\right] .
$$

We impose the anti-bounce-back condition on the boundary :

$$
f_{1}\left(x_{b}, t+\Delta t\right)=-f_{2}\left(x_{e}, t+\Delta t\right)+\Phi .
$$

Due to the fundamental time iteration of a lattice Boltzmann scheme (8), we can write this relation under the form

$$
f_{1}^{*}\left(x_{e}, t\right)=-f_{2}^{*}\left(x_{b}, t\right)+\Phi .
$$

Using a Taylor development of the moments, we obtain :

$$
\begin{aligned}
f_{1}^{*}\left(x_{e}\right)+f_{2}^{*}\left(x_{b}\right) & =\frac{(2+\zeta)}{3} \rho\left(x_{i}\right)+ \\
& +\Delta t^{2} \lambda^{2} \frac{(2+\zeta)}{72}\left[-3+8 \sigma_{1} \sigma_{2}-8 \sigma_{1}(1-2 \theta)\right] \frac{\partial^{2} \rho}{\partial x^{2}}\left(x_{i}\right)+\mathrm{O}\left(\Delta t^{3}\right)
\end{aligned}
$$

We can precise that $\Phi=\frac{(2+\zeta)}{3} \rho\left(x_{i}\right)$ in the right hand side of (22). Then the conclusion holds if we give a null value for the second order term in the previous relation.

- Remark We have extended our result [6] for a general values of the parameter $\theta$.

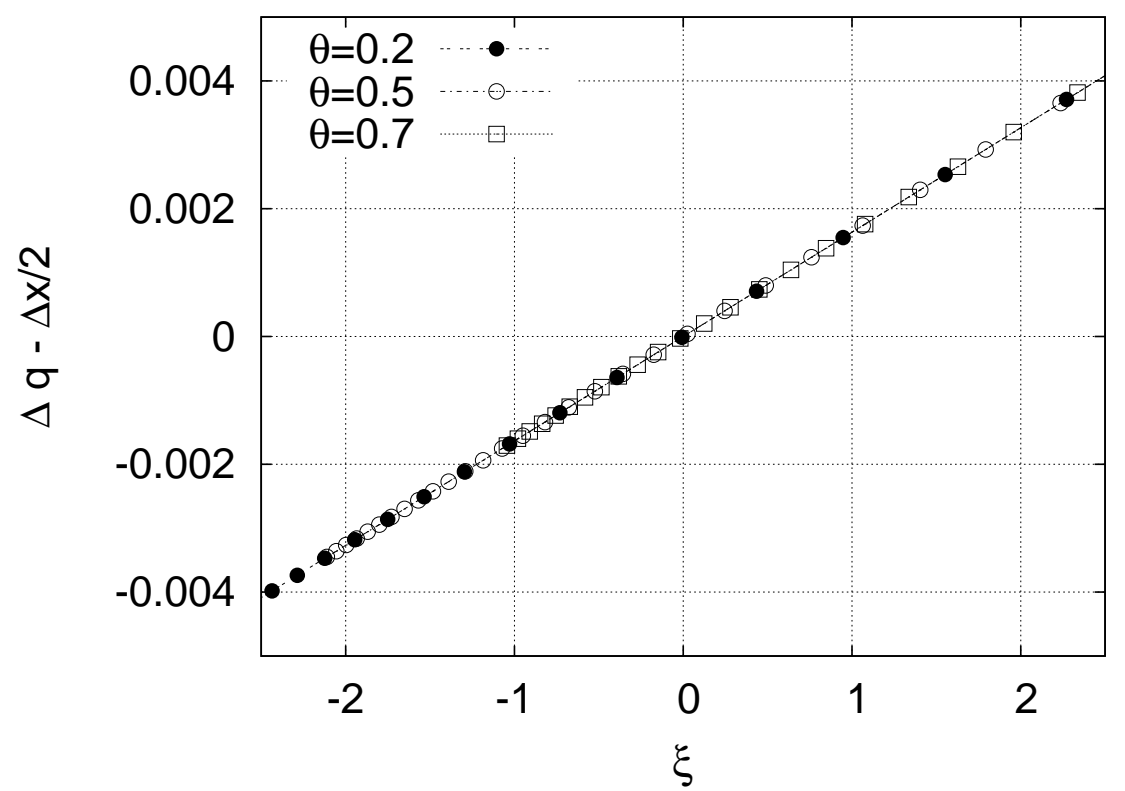

Figure 4. Results of simulations for the solid wall position $\Delta q v s$. the parameter $\xi \equiv-3+$ $8 \sigma_{1} \sigma_{2}-8 \sigma_{1}(1-2 \theta)$ for the D1Q3 scheme for thermal model. We have considered variations of the parameter $\theta$ of the relation (2) for the forcing term $(\theta=0.2$ with bullets, $\theta=0.5$ with circles and $\theta=0.7$ with boxes) and of the parameter $\sigma_{2}$ relative to the last moment. 
François Dubois, Pierre Lallemand and Mohamed-Mahdi Tekitek
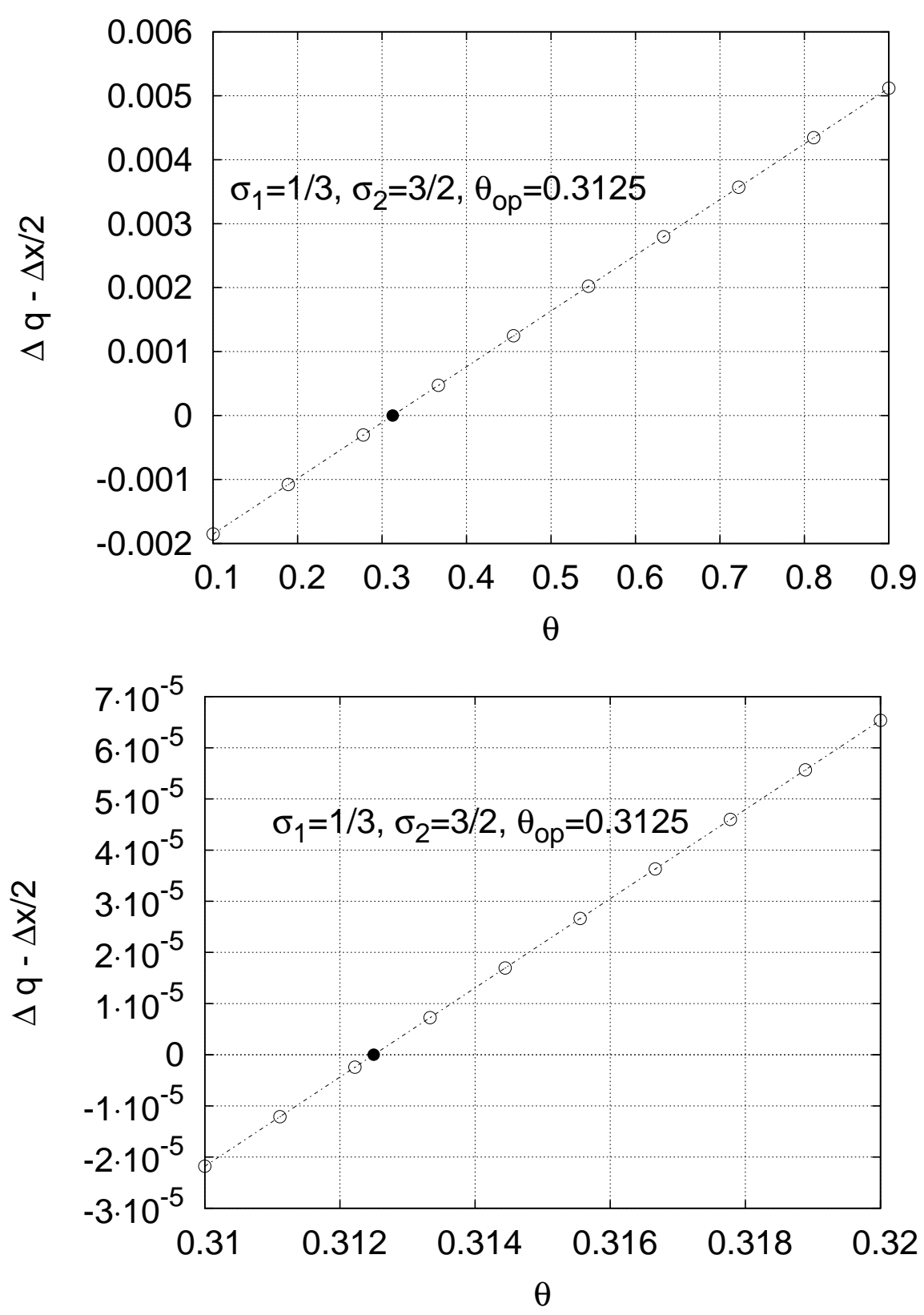

Figure 5. Analogous to the figure 4. On the top: regular variation of the parameter $\theta$ for $\theta \in[0.1,0.9]$. Bottom: zoom of the top figure for $\theta \in[0.31,0.32]$.

\section{4) D2Q9 for a linear acoustic type fluid}

- This scheme is described and analyzed in [14]. The matrix $M$ is now $9 \times 9$. For a fluid model, we have $N=3$ conserved quantities: density $m_{0} \equiv \rho$ and the two components $m_{1} \equiv J_{x}$ and $m_{2} \equiv J_{y}$ of the momentum. The non conserved moments $m_{k}$ for $k \geq 3$ are detailed in [14]. Their equilibrium values are classical:

$$
m_{3}^{e q}=-2 \rho, \quad m_{4}^{e q}=\rho, \quad m_{5}^{e q}=-\frac{J_{x}}{\lambda}, \quad m_{6}^{e q}=-\frac{J_{y}}{\lambda}, \quad m_{7}^{e q}=m_{8}^{e q}=0 .
$$


The relaxation rates are labelled from $s_{3}$ to $s_{8}$ with the two constraints $s_{5}=s_{6}$ and $s_{7}=s_{8}$ to recover an isotropic model compatible with the physics. The particle directions are represented on the figure 1 . With a forcing term of the type $F=\left(0, F_{x}, 0\right)$, the partial equivalent equations at the order 2 are given by

$$
\left\{\begin{array}{l}
\frac{\partial \rho}{\partial t}+\frac{\partial J_{x}}{\partial x}+\frac{\partial J_{y}}{\partial y}=\mathrm{O}\left(\Delta t^{2}\right) \\
\frac{\partial J_{x}}{\partial t}+c_{s}^{2} \frac{\partial \rho}{\partial x}-\zeta \frac{\partial}{\partial x}\left[\frac{\partial J_{x}}{\partial x}+\frac{\partial J_{y}}{\partial y}\right]-v \Delta J_{x}-F_{x}=\mathrm{O}\left(\Delta t^{2}\right) \\
\frac{\partial J_{y}}{\partial t}+c_{s}^{2} \frac{\partial \rho}{\partial y}-\zeta \frac{\partial}{\partial y}\left[\frac{\partial J_{x}}{\partial x}+\frac{\partial J_{y}}{\partial y}\right]-v \Delta J_{y}=\mathrm{O}\left(\Delta t^{2}\right)
\end{array}\right.
$$

where the sound speed $c_{s}$, the shear viscosity $v$ and the bulk viscosity $\zeta$ satisfy respectively $c_{s}^{2}=\frac{\lambda}{3}, v=\Delta t \sigma_{7} \frac{\lambda^{2}}{3}$ and $\zeta=\Delta t \sigma_{3} \frac{\lambda^{2}}{3}$ for the D2Q9 scheme.

- We consider the two-dimensional Poiseuille flow with the D2Q9 lattice Boltzmann scheme. We model this classical problem with the help of an imposed volumic force. Let $\Omega=\left[1, N_{x}\right] \times$ $\left[1, N_{y}\right]$. We enforce periodic condition at the inlet $(i=1)$ and at the outlet $\left(i=N_{x}\right)$. A "bounceback" boundary condition is imposed on the walls to take into account the null velocity $J_{x}=0$ on the upper and lower boundary. A uniform body force $F_{x}$ is applied to model the pressure gradient. We observe that for stationary problems, a lot of time iterations are necessary in order to obtain correct converged results. In that case the lattice Boltzmann method is not the most efficient taking into account the fact that due the exact advection of the lattice the resolution of the stationary problem is not a simple task.

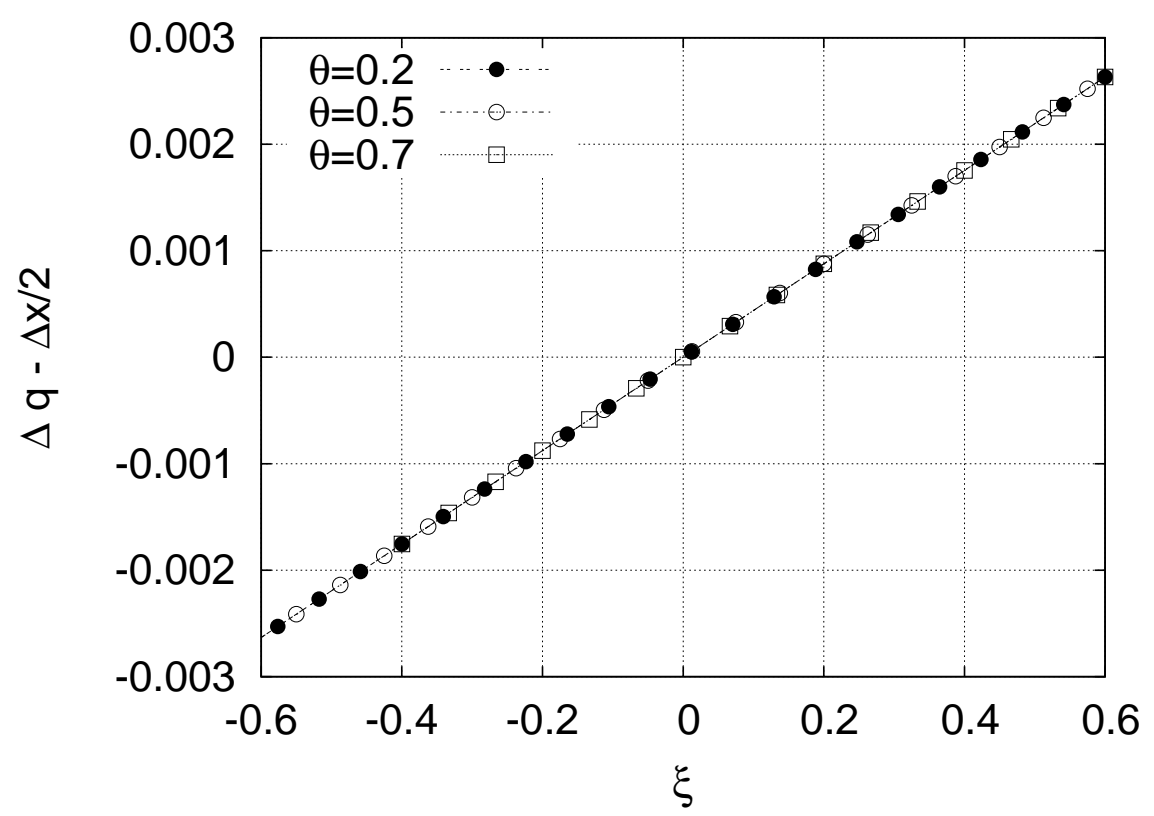

Figure 6. Poiseuille flow for the $\mathrm{D} 2 \mathrm{Q} 9$ scheme. Solid wall position $\Delta q v s$. the parameter $\xi \equiv-3+8 \sigma_{8} \sigma_{5}-8 \sigma_{8}(1-2 \theta)$. Variations of the parameter $\theta$ of the relation (2) for the forcing term ( $\theta=0.2$ with bullets, $\theta=0.5$ with circles and $\theta=0.7$ with boxes $)$ and of the parameter $\sigma_{5}$. 
- Proposition 5. Precise position of the Poiseuille boundary with D2Q9.

With the above choices for the lattice Boltzmann scheme, the solid wall is located at $\Delta q=\frac{\Delta x}{2}$ up to third order when the parameter

$$
\xi \equiv-3+8 \sigma_{8} \sigma_{5}-8 \sigma_{8}(1-2 \theta)
$$

is equal to zero.
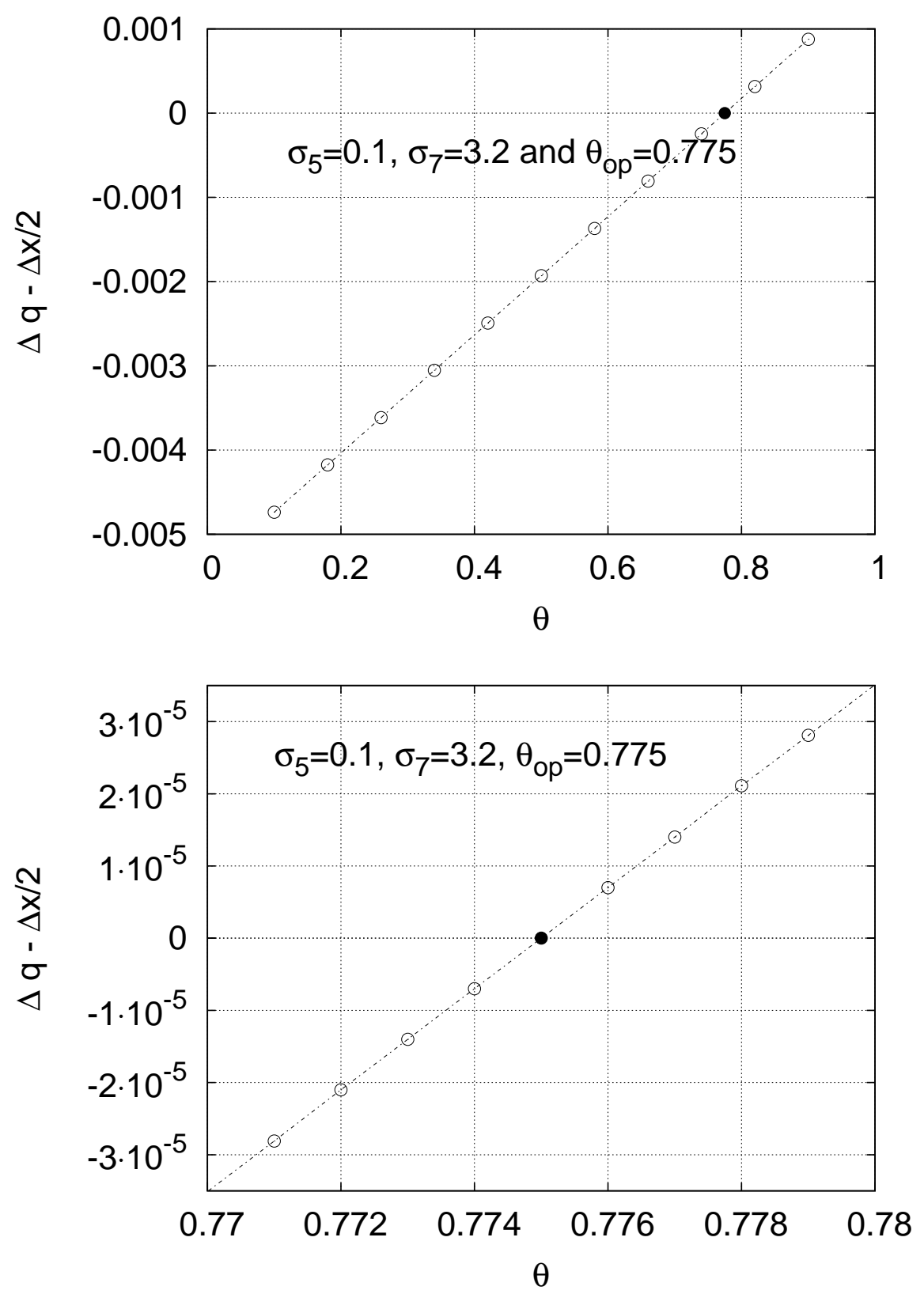

Figure 7. Poiseuille flow with the D2Q9 lattice Boltzmann scheme. Top: solid wall position $\Delta q v s$. the parameter $\xi \equiv-3+8 \sigma_{8} \sigma_{5}-8 \sigma_{8}(1-2 \theta)$. Variations of the parameter $\theta$ of the relation (2) for the forcing term $(\theta=0.2$ with bullets, $\theta=0.5$ with circles and $\theta=0.7$ with boxes) and of the parameter $\sigma_{5}$ relative of the moment $m_{5}$. Bottom: zoom of the top figure, with a variation $\Delta q v s$. the parameter $\theta \in[0.77,0.78]$. 
- The proof of the above Proposition is elementary with traditional algebraic methods (see e.g. [6]) but quite long to develop. We have implemented it without difficulty with a formal computer software. In order to convince the reader, we test the resulting condition $\xi=0$ with appropriate numerical experiments presented in Figure 6 and 7. These experiments extend the ones presented in [6].

\section{5) D2Q13 for a linearized fluid}

- To construct the scheme D2Q13, we add four more velocities to the previous scheme D2Q9 (see the figure 8). The details can be found e.g. in Higuera et al. [10]. Nine moments are analogous to those proposed previously. The equilibrium values are parameterized according to

$$
\left\{\begin{array}{c}
m_{3}^{e q}=a_{1} \rho, m_{4}^{e q}=m_{5}^{e q}=0, m_{6}^{e q}=\frac{c_{1}}{\lambda} J_{x}, m_{7}^{e q}=\frac{c_{1}}{\lambda} J_{y}, \\
m_{8}^{e q}=-\frac{65+63 c_{1}}{24 \lambda} J_{x}, m_{9}^{e q}=-\frac{65+63 c_{1}}{24 \lambda} J_{y}, m_{10}^{e q}=a_{2} \rho, m_{11}^{e q}=a_{3} \rho, m_{12}^{e q}=0 .
\end{array}\right.
$$

In order to simulate isotropic models, the relaxation rates $s_{3}$ to $s_{12}$ satisfy the following relations: $s_{4}=s_{5}, s_{6}=s_{7}$ and $s_{8}=s_{9}$ as proposed e.g. in [2]. With the previous choices, with a forcing term $F=\left(0, F_{x}, 0\right)$ analogous to the one proposed previously for the D2Q9 scheme, the partial equivalent equations take the form (23). In this case the sound speed $c_{s}$, the shear viscosity $v$ and the bulk viscosity $\zeta$ satisfy respectively $c_{s}^{2}=\frac{28+a_{1}}{26} \lambda, v=\Delta t \sigma_{5} \frac{\lambda^{2}}{2}$ and $\zeta=-\Delta t \sigma_{3} \frac{2+a_{1}}{26} \lambda^{2}$. In our compuation we set $a_{1}=-12$. Then $\zeta>0$. We study a Poiseuille flow with the D2Q13 lattice Boltzmann scheme in the framework of an imposed source term. The general choices are analogous to the ones we did with the D2Q9 lattice Boltzmann scheme.

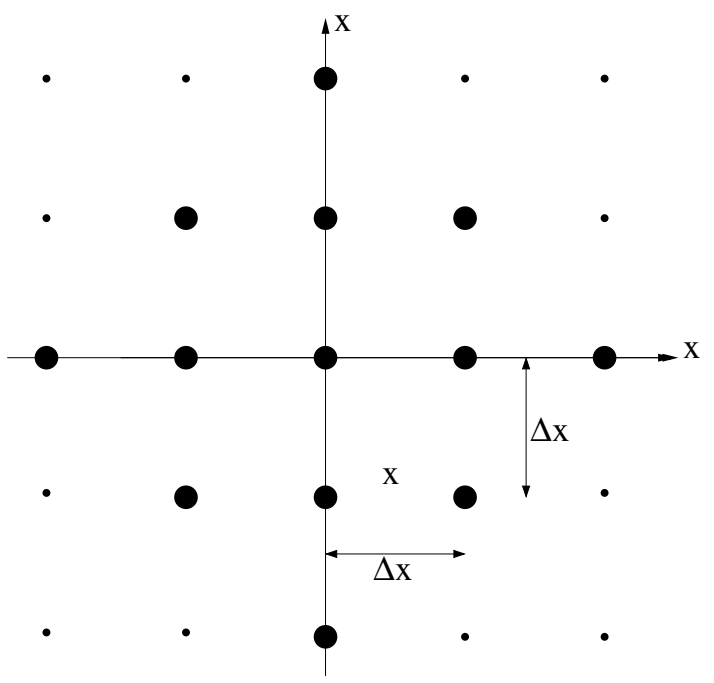

Figure 8. Stencil of the D2Q13 lattice Boltzmann scheme.

- Proposition 6. Precise position of the Poiseuille boundary with D2Q13.

When the parameter

$$
\xi \equiv-7+40 \sigma_{5} \sigma_{7}-8 \sigma_{5}(1-2 \theta)
$$


is null and with the above choices for the lattice Boltzmann scheme, the solid wall is located at $\Delta q=\frac{\Delta x}{2}$ at third order accuracy if and only if $\xi=0$.

This proposition has been solved with the help of formal calculus. We did various experiments analogous to those presented for the D2Q9 scheme. They are summarized in the captions of Figures 9 and 10.

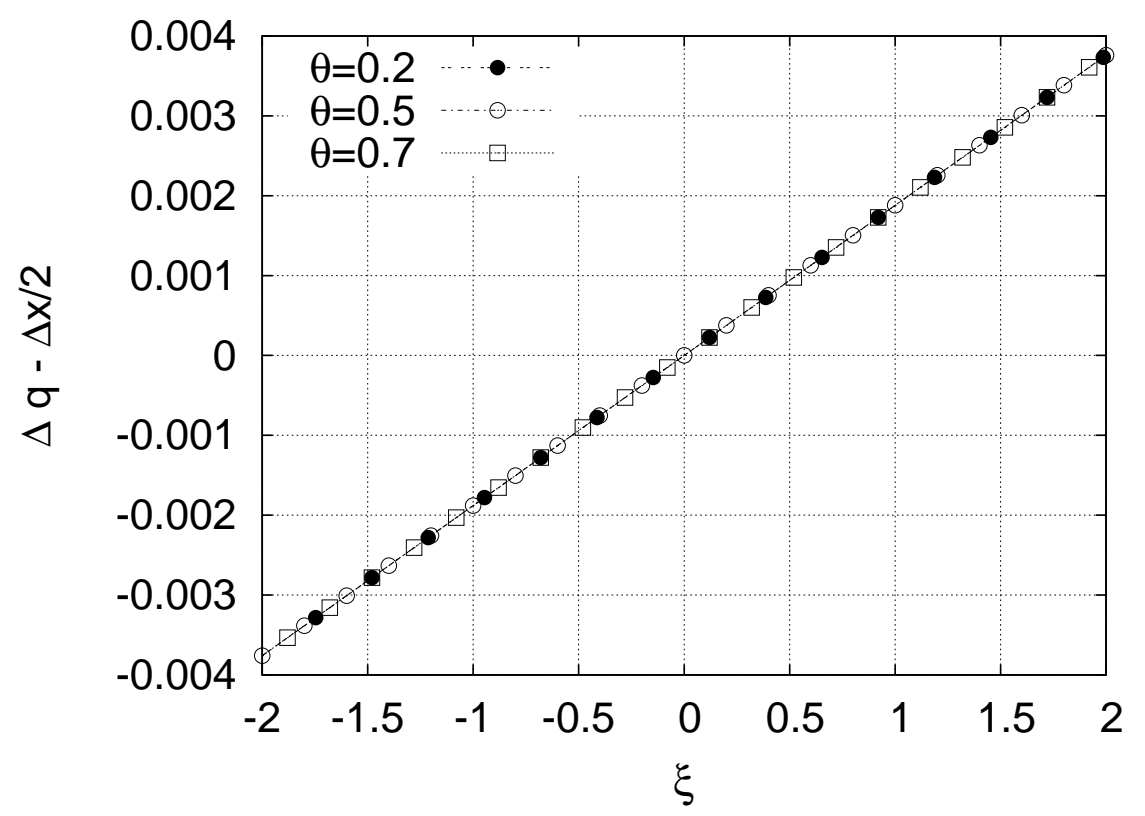

Figure 9. Poiseuille flow for the D2Q13 scheme. Solid wall position $\Delta q v s$. the parameter $\xi \equiv-7+40 \sigma_{5} \sigma_{7}-8 \sigma_{5}(1-2 \theta)$. Variations of the parameter $\theta$ of the relation (2) for the forcing term $(\theta=0.2$ with bullets, $\theta=0.5$ with circles and $\theta=0.7$ with boxes) and of the parameter $\sigma_{5}$.

\section{D2Q9 Conclusion}

- In this contribution, we have extended the "Berliner version" [2] of the formal Taylor expansion method for linear lattice Boltzmann scheme to the case of a possible external force. We have established that the quartic parameters to enforce the precision of the lattice Boltzmann scheme depend not only on the relaxation rates but also on the choice of the parameter $\theta$ for the time integration (2) of the source term.

- We have derived quartic parameters for the D2Q13 model for a Poiseuille flow. Our numerical results validate the formal Taylor development. Nevertheless, a rigorous numerical analysis is still an open question. In future work, we wish to extend the quest of quartic parameters for more general physical problems, study time dependent boundary conditions and give general boundary conditions for any position of the wall relative to the lattice. 
TAYLOR EXPANSION METHOD FOR LINEAR LATTICE BOLTZMANN SCHEMES...
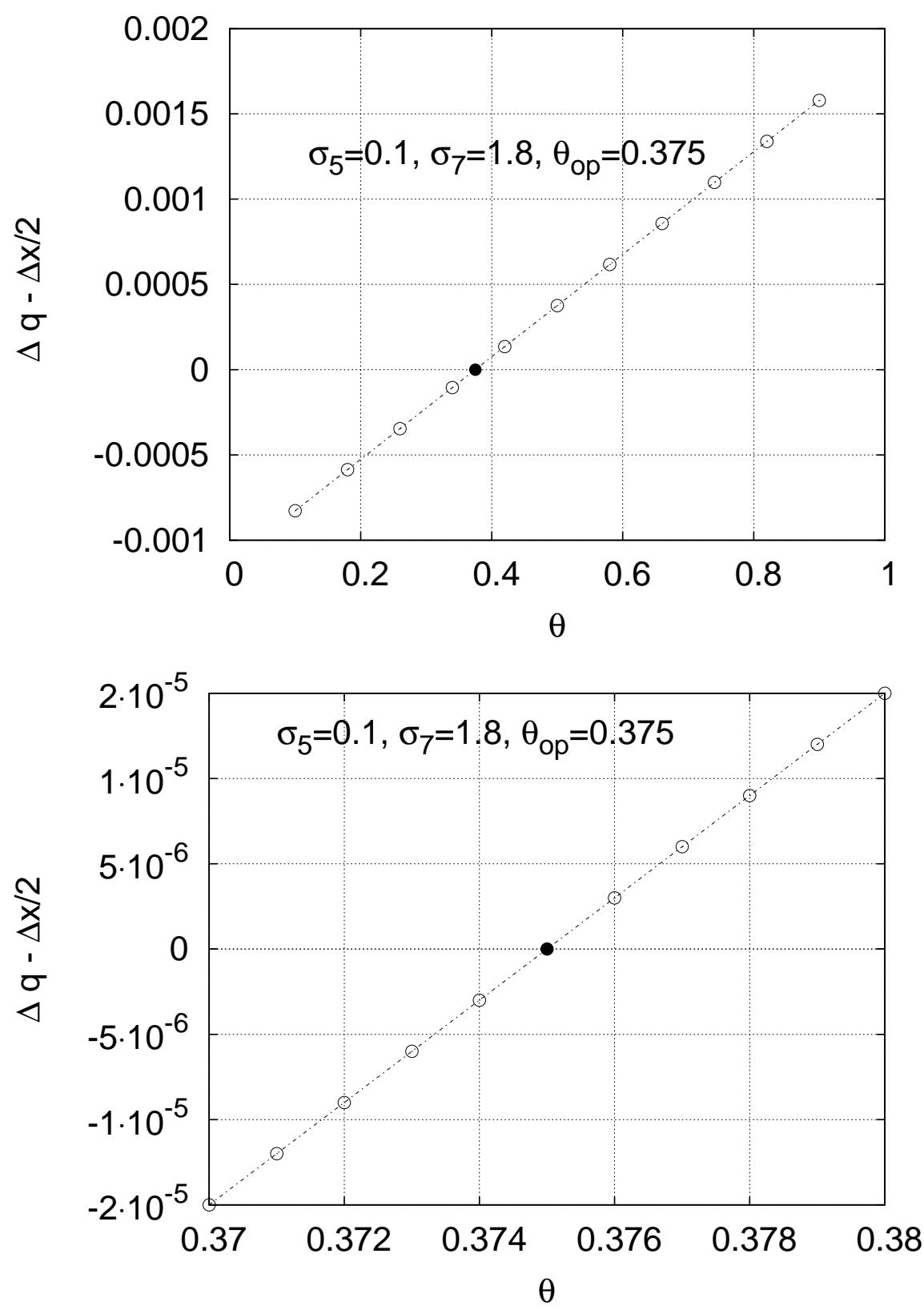

Figure 10. Precise Poiseuille flow with the D2Q13 lattice Boltzmann scheme. Solid wall position $\Delta q v s$. the parameter $\theta$ for the forcing term in (2). Top: $\theta \in[0.1,0.9]$, bottom: zoom of the top figure with $\theta \in[0.37,0.38]$.

\section{Aknowledgements}

The authors thanks the referee for helpful questions after the first draft of this contribution. 
François Dubois, Pierre Lallemand And Mohamed-Mahdi Tekitek

\section{References}

[1] P. Asinari, T. Ohwada, Connection between kinetic methods for fluid-dynamic equations and macroscopic finite-difference schemes, Computers and Mathematics with Applications, vol 58, p. 841-861, (2009).

[2] A. Augier, F. Dubois, B. Graille, P. Lallemand, On rotational invariance of lattice Boltzmann schemes, Computers and Mathematics with Applications, in press,(2013).

[3] M. Bouzidi, M. Firdaous, P. Lallemand, Momentum transfer of a Boltzmann-lattice fluid with boundaries, Physics of Fluids, vol. 13, p. 3452-3459, (2001).

[4] J.M. Buick, C.A. Greated, Gravity in a lattice Boltzmann model, Phys. Rev., vol. 61,

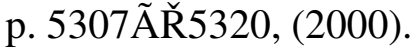

[5] F. Dubois, Equivalent partial differential equations of a lattice Boltzmann scheme, Computers and Mathematics with Applications, vol. 55, p. 1441-1449, (2008).

[6] F. Dubois, P. Lallemand, M.M. Tekitek, On a superconvergent lattice Boltzmann boundary scheme, Computers and Mathematics with Applications, vol. 59, p. 2141-2149, (2010).

[7] I. Ginzburg, P.M. Adler, Boundary flow condition analysis for the three-dimensional lattice Boltzmann model, Journal de Physique II, vol. 4, p. 191-214, (1994).

[8] I. Ginzburg, F. Verhaeghe, D. d'Humières, Two-relaxation-time lattice boltzmann scheme: about parametrization, velocity, pressure and mixed boundary conditions, Communications in Computational Physics, vol. 3, p. 519-581, (2008).

[9] Z. Guo, C. Zheng, B. Shi, Discrete lattice effects on the forcing term in the lattice Boltzmann method, Physical Review E, vol. 65, p. 1-6, (2002).

[10] F. J. Higuera, S. Succi, R. Benzi, Lattice Gas Dynamics with Enhanced Collisions, EuroPhysics Letters, vol. 9, p. 345-349, (1989).

[11] D. d'Humières, Generalized Lattice-Boltzmann Equations, in Rarefied Gas Dynamics: Theory and Simulations (Eds B.D. Shizgal and D.P. Weave), vol. 159 of AIAA Progress in Aeronautics and Astronautics, p. 450-458, (1992).

[12] D. d'Humières, I. Ginzburg, Multi-reflection boundary conditions for lattice Boltzmann models, Physical Review E, vol. 68, (2003).

[13] M. Junk, A. Klar, L.-S. Luo, Asymptotic analysis of the lattice Boltzmann equation. Journal of Computational Physics, vol. 210, p. 676-704, (2005).

[14] P. Lallemand, L.-S. Luo, Theory of the lattice Boltzmann method: Dispersion, dissipation, isotropy, Galilean invariance, and stability, Physical Review E, vol. 61, p. 6546-6562, (2000). 
[15] A. Lerat, R. Peyret, Noncentered Schemes and Shock Propagation Problems, Computers and Fluids, vol. 2, p. 35-52, (1974).

[16] L.-S. Luo, Unified theory of lattice Boltzmann models for non-ideal gases, Phys. Rev. Lett., vol. 81, p. 1618-1621, (1998).

[17] N.S. Martys, X. Shan, H. Chen, Evaluation of the external force term in the discrete Boltzmann equation, Phys. Rev. E, vol. 58, p. 6855-6857, (1998).

[18] A.A. Mohamad, A. Kuzmin, A critical evaluation of force term in lattice Boltzmann method, natural convection problem, International Journal of Heat and Mass Transfer, vol. 53, p. 990 Ã ̌̌ 996, (2010).

[19] Y.H. Qian, D. d'Humières, P. Lallemand, Lattice BGK Models for Navier-Stokes Equation, EuroPhysics Letters, vol. 17, p. 479-484, (1992).

[20] S. Succi, R. Benzi, F. Higuera, Lattice Gas Methods for Homogeneus and Inhomegeneus Hydrodynamics, Discrete kinetic theory, lattice gas dynamics and foundations of hydrodynamics, R. Monaco Editor, World Scientific Publishing, Singapore, p. 329-342, (1989).

[21] R.F. Warming, B.J. Hyett, The modified equation approach to the stability and accuracy analysis of finite difference methods, Journal of Computational Physics, vol. 14, p. 159$179,(1974)$. 